OPEN ACCESS

Edited by:

Fabio Grizzi,

Humanitas Clinical and Research

Center, Italy

Reviewed by: Shashwat Sharad,

Uniformed Services University of Health Sciences, USA

Sanja Štifter,

University of Rijeka, Croatia

${ }^{*}$ Correspondence:

Colleen Bailey

colleen.bailey@ucl.ac.uk

tThese authors have contributed equally as first authors.

Specialty section:

This article was submitted to Genitourinary Oncology, a section of the journal

Frontiers in Oncology

Received: 23 January 2017 Accepted: 08 March 2017

Published: 24 March 2017

Citation:

Bourne RM, Bailey C, Johnston EW,

Pye $H$, Heavey S, Whitaker $H$,

Siow B, Freeman A, Shaw GL,

Sridhar A, Mertzanidou T,

Hawkes DJ, Alexander DC,

Punwani S and Panagiotaki E (2017) Apparatus for Histological Validation

of In Vivo and Ex Vivo Magnetic

Resonance Imaging of the Human Prostate.

Front. Oncol. 7:47. doi: 10.3389/fonc.2017.00047

\section{Apparatus for Histological Validation of In Vivo and Ex Vivo Magnetic Resonance Imaging of the Human Prostate}

\author{
Roger M. Bourne ${ }^{1 t}$, Colleen Bailey ${ }^{2 * t}$, Edward William Johnston ${ }^{3}$, Hayley Pye \\ Susan Heavey ${ }^{4}$, Hayley Whitaker, Bernard Siow 5 , Alex Freeman ${ }^{6}$, Greg L. Shaw ${ }^{7,8}$, \\ Ashwin Sridhar ${ }^{7,8}$, Thomy Mertzanidou', David J. Hawkes², Daniel C. Alexander', \\ Shonit Punwani ${ }^{3}$ and Eleftheria Panagiotaki ${ }^{2}$

\begin{abstract}
Discipline of Medical Radiation Sciences, Faculty of Health Sciences, University of Sydney, Sydney, NSW, Australia, ${ }^{2}$ Centre for Medical Image Computing, University College London, London, UK, ${ }^{3}$ Centre for Medical Imaging, University College London, London, UK, ${ }^{4}$ Centre for Molecular Intervention, University College London, London, UK, ${ }^{5}$ Centre for Advanced Biomedical Imaging, University College London, London, UK, ${ }^{6}$ Department of Research Pathology, University College London, London, UK, ' Division of Surgery and Interventional Science, University College London,
\end{abstract} \\ London, UK, ${ }^{8}$ Department of Urology, University College London Hospitals, London, UK
}

This article describes apparatus to aid histological validation of magnetic resonance imaging studies of the human prostate. The apparatus includes a 3D-printed patientspecific mold that facilitates aligned in vivo and ex vivo imaging, in situ tissue fixation, and tissue sectioning with minimal organ deformation. The mold and a dedicated container include MRI-visible landmarks to enable consistent tissue positioning and minimize image registration complexity. The inclusion of high spatial resolution ex vivo imaging aids in registration of in vivo MRI and histopathology data.

Keywords: prostate, prostate cancer, MRI validation, in vivo MRI, ex vivo MRI, histology, co-registration, fixation

\section{THE NEED FOR HISTOLOGICAL VALIDATION OF MRI}

Imaging provides valuable non-invasive information for diagnosis and treatment of prostate cancer. The current state of the art is multi-parametric MRI (mp-MRI) (1), although even this lacks specificity and cannot provide reliable grading information. Advanced techniques $(2,3)$ show promise for probing cancer microstructure and may be more specific than conventional methods. However, rigorous validation is needed to assess the current and potential value of such techniques in prostate cancer management. The current gold standard for validation is histological assessment of whole mount serial sectioned radical prostatectomy specimens, but comparing information from such disparate images presents several challenges.

Below we give an overview of these challenges and the current methods for addressing some of them. Then, we describe a mold-based apparatus and imaging protocol that include ex vivo imaging and a number of innovations that improve alignment of imaging and histology planes and minimize in-plane rotational differences to improve the quality of the $2 \mathrm{D}$ registration processes. The apparatus also allows for the collection of supplementary ex vivo MR data for both the fresh unfixed and the fixed prostate specimen. 


\section{PROBLEMS IN HISTOLOGICAL VALIDATION OF MRI METHODS}

There are a number of major technical problems complicating any attempt to directly match and correlate MRI and histology data (4). These difficulties include

- Unmatched tissue planes. In practice there is usually no direct coordination of presurgical imaging methods and surgical specimen processing. This results in MRI slice planes usually having different plane orientation and plane spacing from the histology images with no clear correspondence between the two so that, at best, only a qualitative correlation of MRI and pathology data is feasible.

- Unmatched spatial resolution. A volume element ("voxel”) in prostate MRI (the source of each semi-discrete item of measurement data) has a typical size that may vary from $0.2 \mathrm{~mm} \times 0.2 \mathrm{~mm}$ in-plane by $1 \mathrm{~mm}$ slice thickness to $2 \mathrm{~mm} \times 2 \mathrm{~mm}$ in-plane by $5 \mathrm{~mm}$ slice thickness. By contrast, a typical histological image used for pathology assessment is based on a 3-5 $\mu \mathrm{m}$ thick tissue section and has an in-plane resolution $<1 \mu \mathrm{m}$. Even under highly idealized conditions, where the histology and MRI slices are "co-planar," the MRI signal originates from a much larger tissue volume than is represented in the histology slice. Thus, there may be tissue structure heterogeneity within the MRI slice that is not reflected in the histological data.

- Tissue deformations. The prostate is deformed in vivo by voluntary and involuntary body movements, bowel contents, and by the imaging system itself if an endorectal coil is employed for signal detection. Upon resection, the prostate is detached from its supporting tissues and associated vasculature, resulting in shape changes due to removal of mechanical tension and compression and hemodynamic pressure (5). Dehydration and embedding of the tissue during processing for histology results in tissue shrinkage and thin sectioning may cause further deformations.

- Unmatched image features. MRI and histological staining produce image contrast according to very different tissue properties. The features present in MR images may not be easily identified in histology images and vice versa, leading to a difficulty in assessing the accuracy of any co-registration process.

\section{SURVEY OF METHODS}

The validation problems mentioned earlier have been addressed with varying degrees of completeness by various methods, some of which have been reviewed in Ref. $(4,6)$. Here, we provide a brief overview of the approaches and problems addressed by the method we describe.

A partial 3D histology "map" can be reconstructed from a stack of histology slices and registered with MR images by a 3D process $(7,8)$; however, the accuracy of this approach is severely limited by the low out of plane resolution of the MRI data. The problem of missing inter-plane histology data can be addressed by manual $(9,10)$ or automatic (11) selection of the most closely aligned histology and image planes. Ideally, this reduces the problematic $3 \mathrm{D}$ registration to a more tractable $2 \mathrm{D}$ process. As the accuracy of this approach is highly dependent on co-alignment of imaging planes with histology sections, a number of methods have been described based on production of a patient-specific $3 \mathrm{D}$-printed mold, the shape of which is defined by the in vivo imaging data. The aim of the mold is to hold the prostate in the same conformation in which it was imaged while guides in the mold align the tissue cutting planes with the image slice positions (12-15). The precision of these mold-based methods is dependent on the degree to which the mold matches the shape of the excised prostate specimen and the amount of any mispositioning or rotation of the prostate in the mold. Rotation errors are more likely around the axes in which the specimen has greatest rotational symmetry. Inclusion of a urethral catheter in the specimen and mold design can decrease rotation errors about the left-right axes of the prostate (6).

High spatial resolution ex vivo imaging of the prostate specimen has been used as an intermediate "stepping-stone" to improve the accuracy of co-registration of in vivo MRI and histology data, both with (6) and without (16) a patient-specific mold. However, the mold design can be improved to better facilitate comparison between MR and histological images.

\section{OUTLINE OF IMAGING AND SPECIMEN HANDLING}

The following points outline the method of imaging and construction of a patient-specific 3D-printed mold to optimize physical location of the prostate in in vivo MR images, ex vivo MR images, and histology images. The aim is to reduce the inherent 3 -dimensional co-registration problem to a more tractable and precise 2-dimensional process.

\subsection{In Vivo Imaging}

This study was carried out in accordance with the recommendations of the UK Research Governance Framework version 2, UK Research Ethics Committee with written informed consent from all subjects and approved by the NRES Committee London-Surrey Borders (REC 15/LO/0692). All subjects gave written informed consent in accordance with the Declaration of Helsinki.

The geometry for the in vivo imaging needs to be compatible with the local clinical histopathology processing and reporting protocol. In our case, the pathology department uses $5 \mathrm{~mm}$ thick sections cut approximately transaxial to the prostatic urethra and perpendicular to the posterior face of the prostate. We perform T2-weighted imaging with $2.5 \mathrm{~mm}$ slice thickness (with no gap or $2.5 \mathrm{~mm}$ gap) in the "true axial" scanner XY plane. The central slice is centered approximately mid-organ and is defined as the reference for all subsequent imaging and processing and is defined by MR visible landmarks in the patient-specific mold.

For the data illustrating the methods in this paper mp-MRI was performed on a 3-T scanner (Achieva, Philips, Best, the Netherlands), using pelvic phased array coils. The $0.2 \mathrm{mg} / \mathrm{kg}$ (up to $20 \mathrm{mg}$ ) of a spasmolytic agent (Buscopan; Boehringer 
Ingelheim, Germany) was administered intravenously prior to imaging to reduce bowel peristalsis. mp-MRI comprises axial and coronal T2 turbo spin echo (TSE) imaging, supplemented with diffusion-weighted imaging at $b$-values $0,150,500$, and $1,000 \mathrm{~s} / \mathrm{mm}^{2}$. A dynamic contrast enhanced (DCE) acquisition was subsequently performed using spoiled gradient echo with fat saturation and a 12 -s time resolution. Intravenous contrast agent $(0.2 \mathrm{~mL} / \mathrm{kg}$; Prohance, Bracco, Milan, Italy) was injected at the beginning of the 6 th acquisition at $3 \mathrm{~mL} / \mathrm{s}$ followed by $20 \mathrm{~mL}$ of saline.

\subsection{Contouring of In Vivo Images for Patient-Specific Mold Specification}

MR datasets were analyzed using Osirix Version 7.0 (Bernex, Switzerland). A board certified Radiologist (Edward William Johnston) manually contoured the entire prostate (from base to apex) using the closed polygon tool on high-resolution axial T2-weighted images. Contoured landmarks comprise the hypointense prostate capsule and the anterior fibromuscular stroma where the capsule is absent anteriorly. The periprostatic fat, neurovascular bundles, seminal vesicles, distal urethral sphincter, and bladder were not included inside the contoured volume but provided useful information as to the extent of the prostate. The positions of contours were checked in all planes and adjusted accordingly until their appearance was satisfactory. Where available, at least 3 slices of coronal and/or sagittal images were contoured at the center of the gland to corroborate axial contours, but the edge of the prostate was not contoured as it is poorly delineated in these planes. To estimate urethral catheter position, the urethra was demarcated where visible using axial T2-weighted images and interpolating based on normal anatomy and adjacent slice position where invisible.

\subsection{Prostatectomy and Specimen Preparation}

The radical prostatectomy specimen was collected immediately upon resection to minimize ischemia time and taken to the pathology department without formalin fixation. The fresh specimen was inked and dried, and the seminal vesicles and any metal clips that would cause magnetic susceptibility artifacts were removed. A 4.2-mm diameter silicon rubber catheter was inserted through the urethra, the prostate placed into the mold, and the mold halves fastened with four plastic cable ties. The specimen inside the closed mold was then inserted into the canister, which had been pre-filled with saline. Gentle agitation was used to eliminate air bubbles, and then the sealing piston was inserted fully to ensure alignment of the mold reference plane with the external reference landmarks. Excess fluid was ejected through the vent, which was then capped.

\subsection{Ex Vivo Prostate Imaging}

Fresh ex vivo scanning was performed on both a 3-T clinical MRI scanner (Philips Achieva, Best, the Netherlands) and a 9.4-T 20-cm horizontal bore MRI (Varian Inc., Palo Alto, CA, USA). Fixed imaging was conducted only at 9.4 T. The reference plane landmarks on the exterior of the mold canister (see section 6 below) were used to position the reference slice of the sample near the isocenter of the magnet.

For 3-T MRI, the sample was positioned at the center of an 8-channel knee coil (Philips, Best). T2-weighted TSE images $(\mathrm{TE}=100 \mathrm{~ms}$, echo train length $=16, \mathrm{TR}=5.2 \mathrm{~s}$, field of view $18 \mathrm{~cm} \times 18 \mathrm{~cm}$ ) were acquired with $0.4 \mathrm{~mm} \times 0.4 \mathrm{~mm}$ resolution in-plane, $2 \mathrm{~mm}$ slice thickness, and $0.5 \mathrm{~mm}$ slice gap.

Imaging at $9.4 \mathrm{~T}$ was performed using $400 \mathrm{mT} / \mathrm{m}$ gradients and a $72 \mathrm{~mm}$ internal diameter quadrature coil (RAPID Biomedical, Rimpar, Germany). A multi-slice gradient echo sequence $(\mathrm{TE}=5 \mathrm{~ms}, \mathrm{TR}=75 \mathrm{~ms}$, field of view $8 \mathrm{~cm} \times 8 \mathrm{~cm}$ ) was acquired giving $0.625 \mathrm{~mm} \times 0.625 \mathrm{~mm}$ in-plane resolution, $1 \mathrm{~mm}$ slice thickness, and no slice gap. These images were used to locate the reference slice using the reference plane landmarks (see section 5.1 below), as shown in Figure 1. The same procedure for reference slice location was repeated following specimen fixation.

\section{MOLD DESIGN AND 3D PRINTING}

\subsection{Mold Template}

The mold template is $62 \mathrm{~mm}$ diameter, designed to fit inside a $63.5 \mathrm{~mm}$ internal diameter canister that contains the prostate immersed in saline during ex vivo imaging. The mold design is based on the abilities and constraints of nylon powder printing (selective laser sintering using EOSINT P100 with PA2200 powder; 40-50 $\mu \mathrm{m}$ grain size). Note that the mold design described here could not be produced on a typical low-cost plastic filament printer due to the lower dimensional precision and the need for these devices to print support material that would be extremely difficult to remove from the design we describe. A more basic mold design suitable for a filament printer is described in Ref. (6). The mold features are illustrated in Figure 2 and include

- A chamfer at one end of the mold indicates the apex end of the prostate, so that orientation of the prostate during ex vivo imaging is as for in vivo imaging with the patient lying in feetfirst supine position.

- LP. Two 4-mm diameter locator pins ensure alignment of the mold halves.

- CP. Two 0.4-mm wide slots, spaced $5.0 \mathrm{~mm}$ apart, define the cutting planes for organ sectioning.

- B. 0.5-mm diameter braces stabilize the mold shape during ex vivo imaging. These are removed immediately prior to organ sectioning.

- RPLM. Reference plane land marks (1.5-mm diameter axial channels) are filled with liquid when the prostate and mold are inserted in the imaging canister and provide MR visible landmarks that enable precise matching of in vivo and ex vivo imaging planes. Relative to the cutting planes the landmarks are offset toward the apex of the prostate to bring the center of the MRI planes into closer alignment with the histology sections that are routinely cut from the apex face of the tissue blocks.

- U. A 4.2-mm diameter saline-filled silicon rubber urethral catheter is inserted in the prostate with curvature and 


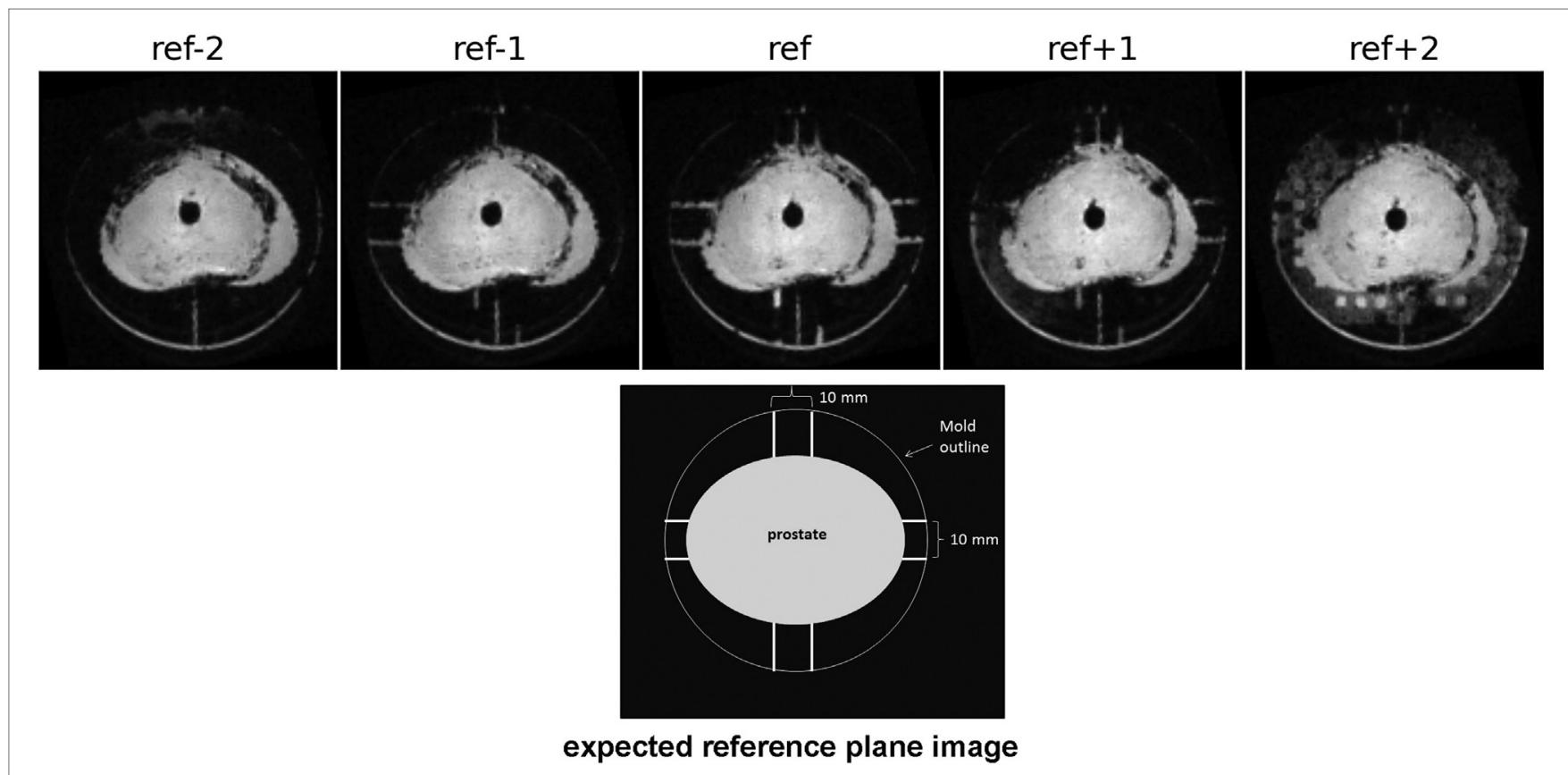

FIGURE 1 | Images acquired at $1 \mathrm{~mm}$ spacing demonstrate the use of the reference plane landmark channels (some of which are obscured by air bubbles here) to locate the reference slice. When correctly positioned and aligned the reference image will show the water filled channels on the A/P and L/R sides of the prostate.

orientation defined during contouring of the in vivo images. The catheter assists in constraining any rotations of the prostate in the sagittal plane.

- S. Flexible springs printed into the mold press against the wall of the canister and minimize any movement or vibration of the mold in the canister during imaging.

- G. A groove in the top of the mold matches the internal rib in the canister (Figure 3 ) and precludes any rotation of the mold in the axial plane.

- CT. The corners of the mold halves are fastened with four plastic cable ties.

\section{MOLD CONTAINER FOR EX VIVO IMAGING}

For ex vivo imaging (both before and after formalin fixation), the specimen in the mold is constrained inside a plastic canister. A liquid-tight seal is made with a piston that also serves as the mounting base for attachment of the sample to the scanner's small animal bed. Details are described in Figure 3 and below:

- Canister. The canister is $68 \mathrm{~mm}$ outside diameter, $63.5 \mathrm{~mm}$ internal, to fit with $2 \mathrm{~mm}$ clearance inside the $72 \mathrm{~mm}$ internal diameter RF coil used for 9.4-T ex vivo imaging. The canister is printed in rigid photopolymer (Objet, Stratasys), which is non-porous. Landmarks on the external surface of the canister define the position of the reference slice and top midline of the mold. An internal rib fits the groove in the top of the mold and prevents any rotation of the mold inside the canister, thus ensuring minimal axial plane rotational differences between in vivo and ex vivo images (see Figure 2).

- Piston. The canister is sealed liquid tight with a piston (also printed in rigid photopolymer) fitted with three O-rings lubricated with light grease. To immerse the prostate in liquid and minimize inclusion of air bubbles the canister is placed vertically and filled with $200 \mathrm{~mL}$ of saline. The prostate in the mold is inserted, pushed to the bottom of the canister, and agitated to expel air bubbles. The piston is then inserted and excess liquid is expelled into the central well via the vent which is then sealed with a plastic pin. The center line landmark (CL) on the piston is aligned with the center line mark on the canister. The fully inserted piston fixes the mold at the base of the canister, so that the mold reference plane is aligned with the external landmarks. The mold and prostate are thus fixed in a defined position relative to the external landmarks on the canister and piston.

- Mounting bracket. The base of the piston is attached to a mounting bracket that is designed specifically for the small animal bed of the MRI scanner. The bracket is printed on the SLS printer that provides a rigid product (slightly porous so unsuitable for canister and piston).

\section{FORMALIN FIXATION OF PROSTATE IN MOLD}

The porous mold design enables fixation of the prostate in situ. The mold/prostate is removed from the canister and immersed in 1-L fixative solution in a narrow vessel placed on a magnetic stirrer overnight. The low density of the nylon mold provides 

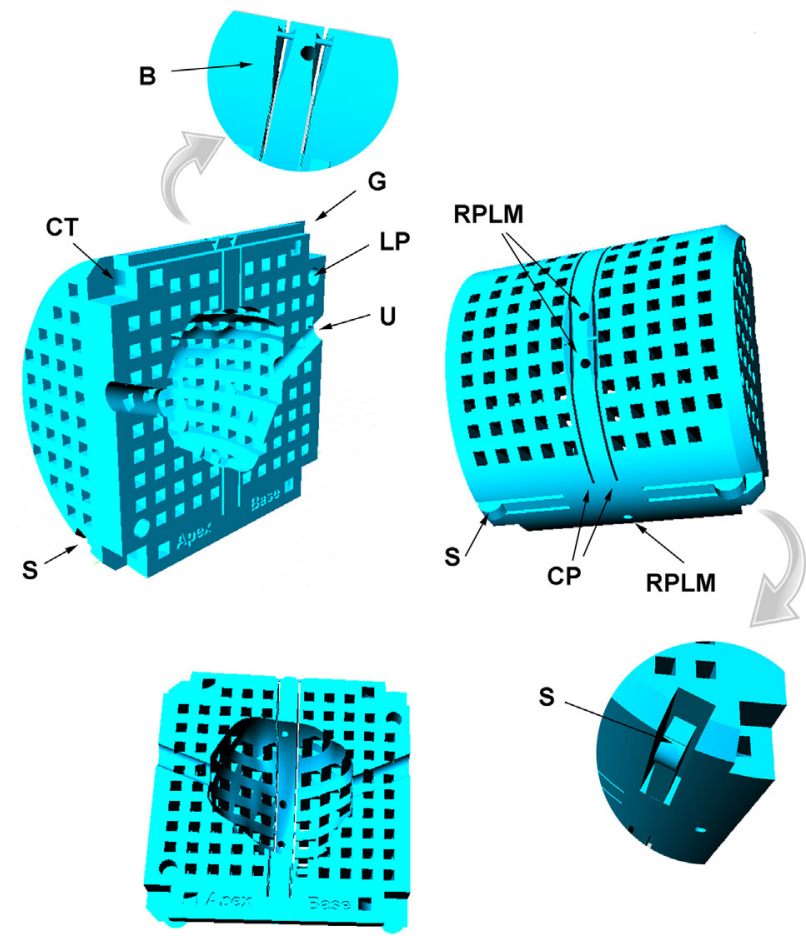

FIGURE 2 | Mold design features. See text for label descriptions.

sufficient buoyancy for the prostate to float just below the liquid surface. Use of the stirrer enhances penetration of the fixative through the mold structure and into the prostate. Post fixation, the immersion solution is replaced with 1 - $\mathrm{L}$ saline for $8 \mathrm{~h}$ to dilute the formalin which, at full strength, severely reduces the sample T2 leading to poor signal quality.

Formalin fixation leads to tissue shrinkage (17) and shape changes (18). Fixation of the prostate in the mold constrains any shape changes.

\section{APPARATUS FOR SECTIONING PROSTATE IN MOLD}

Upon completion of fixation and ex vivo imaging, the prostate is sectioned for histological processing (Figure 4). The mold defines two cutting planes spaced $5 \mathrm{~mm}$ apart on either side of the imaging reference plane. Prior to cutting, the mold stabilizer braces (Figure 2) are removed with a scalpel blade, and the mold placed in a dedicated cradle $(C)$. The two cuts on either side of the reference slice are made simultaneously using a pair of skin graft blades (R55170, Rocket Medical, Washington, UK) mounted in an SLS-printed handle $(\mathrm{H})$. The handle and a detachable spacer (S) maintain the blades at 5-mm spacing that aids insertion of the blades into the slots in the top of the mold.

After cutting of the reference plane section, the mold is opened and the prostate removed. The base and apex volumes are then progressively sectioned at $5-\mathrm{mm}$ thickness using a flat plate with 5 - $\mathrm{mm}$ high rails to guide the blade.

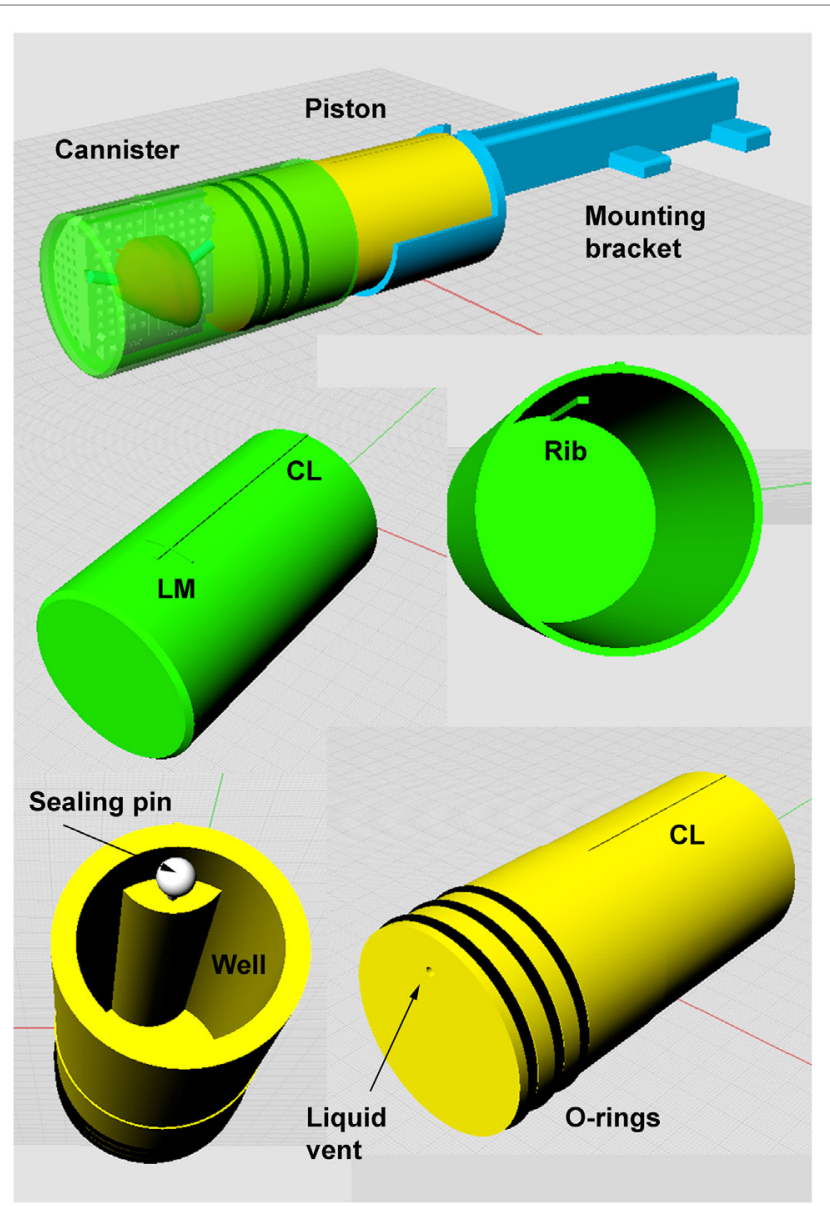

FIGURE 3 | Apparatus for ex vivo imaging of the prostate immersed in saline. See text for design features description.

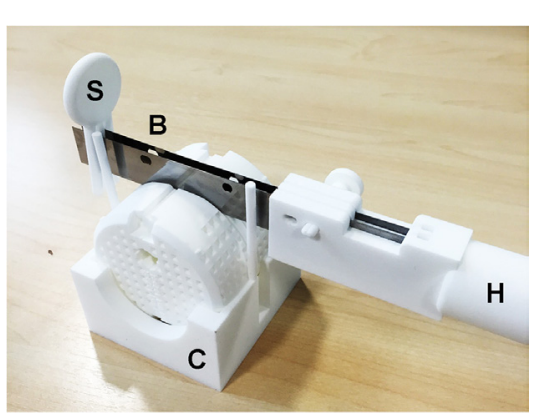

FIGURE 4 | Prostate slicing in mold. The mold is stabilized in a cradle (C). The handle $(\mathrm{H})$ and separate spacer $(\mathrm{S})$ maintain two skin graft blades $(B)$ at the same $5 \mathrm{~mm}$ spacing as the mold cutting planes.

Whole mount tissue sections are processed according to standard laboratory protocol. These sections are cut from the prostate apex face of each block. Note that the reference plane landmarks in the mold are offset toward the apex to account for the histology slice position typically being closer to the apex side of each tissue section than to the base side. 


\section{IMAGE REGISTRATION}

There is a very wide range of $2 \mathrm{D}$ image registration methods that could be applied for alignment of the MRI and histology images. As the focus of this paper is on the apparatus used for prostate imaging and sectioning, we present here only an outline of the methods used to produce the example data presented in the figures.

Hematoxylin and eosin stained whole mount sections were digitally scanned with a $20 \times$ objective (Hamamatsu NanoZoomer). Histological images were downsampled to $0.25 \times$ and converted to grayscale for registration. The high-resolution ex vivo T2 MRI slice for registration was selected based on the mold landmarks. Registration to the corresponding histological slice used an intensity-based $2 \mathrm{D}$ rigid registration based on a block-matching strategy $(19,20)$ with the correlation coefficient as similarity measure. The ex vivo MRI was registered to the in vivo MRI using $2 \mathrm{D}$ affine registration, restricting the block matching in the in vivo image to the prostate region using the contour from section 4.2 .

\section{SAMPLE RESULTS}

An example case is shown in Figure 5.

Registered MRI and histology slices are shown in Figure 6. Slicing artifacts, particularly near the urethra, are visible and may be corrected by additional non-rigid registration techniques. Nevertheless, the spatial correspondence between the ex vivo and histology images can be seen, including in the peripheral zone shape and in the outline of glandular regions within the transition zone. The peripheral zone partially collapses between in vivo and ex vivo imaging, but internal structures within the transition zone show correspondence.

\section{LIMITATIONS AND FUTURE DIRECTIONS}

The apparatus detailed here addresses many of the issues described in section 2 and extends previous methods by improving the alignment of in vivo and ex vivo imaging planes with each other and with histopathology sections. Nevertheless, a number of problems remain

- Prostate contouring from in vivo images. Contour delineation on the in vivo images aims to trace the expected surgical margins. These are normally close to the prostatic capsule but may vary case by case and the capsule is absent around the prostatic apex. Depending on the T2 image quality and characteristics of the individual prostate, it is sometimes difficult to discriminate the capsule from adjacent intra and extra-prostatic structures. One strategy to accommodate these uncertainties is to print multiple versions of the mold
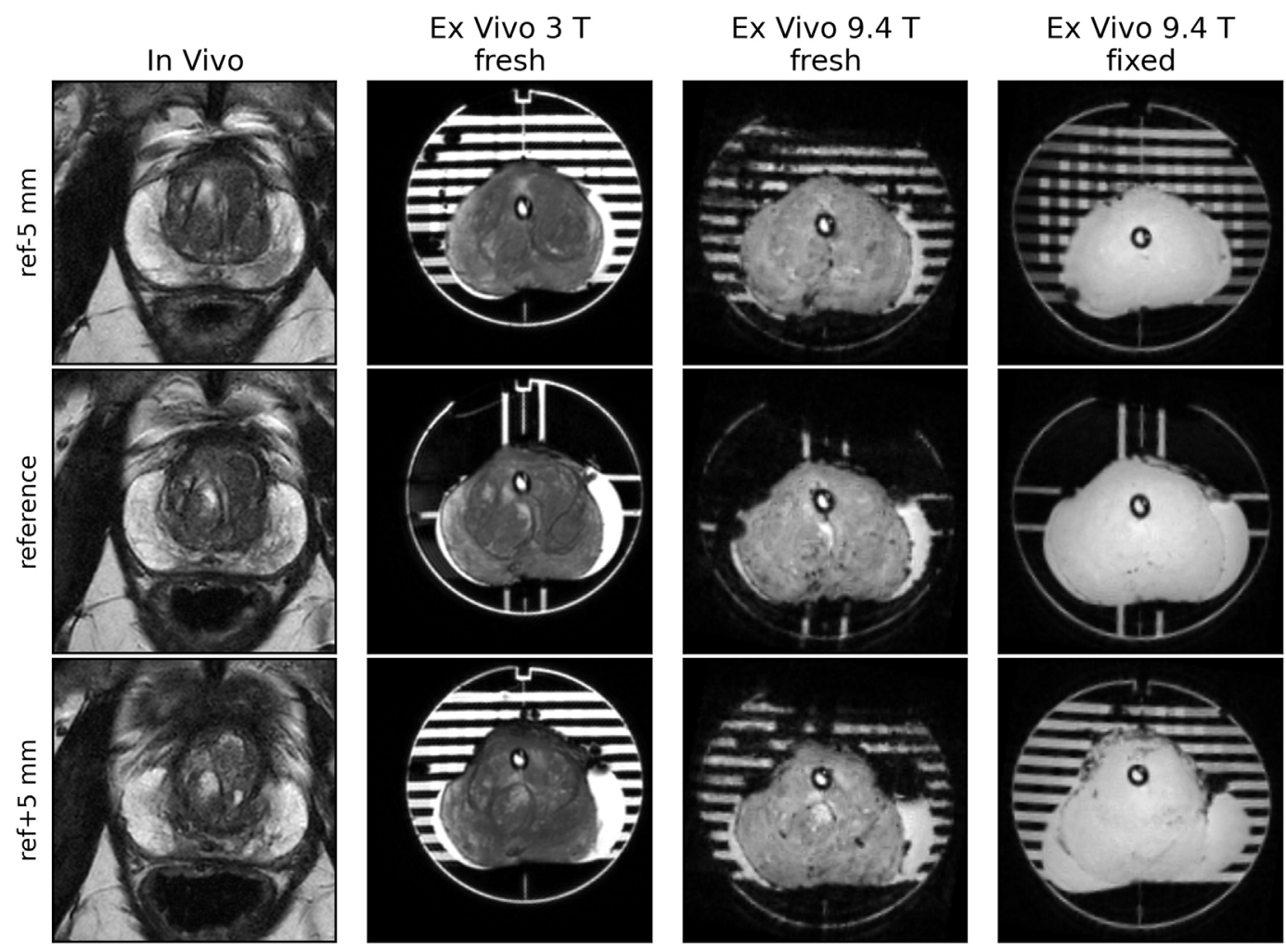

FIGURE 5 | Images from the reference slice and slices $\mathbf{\pm 5} \mathbf{~} \mathbf{m m}$ from the reference. Contours from the in vivo scan's reference slice were aligned with the reference plane of the mold. The 8 channels in the reference plane were used to locate the reference slice for fresh scans at 3 and 9.4 , as well as after fixation. 

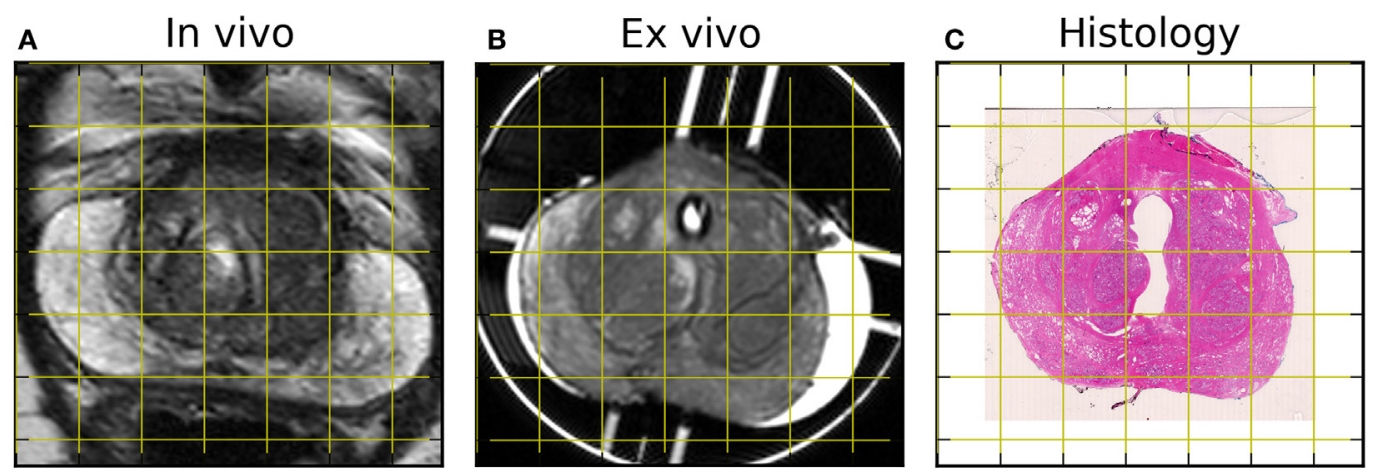

FIGURE 6 | Sample in vivo (A) and ex vivo (B) MR images after rigid registration to hematoxylin and eosin-stained histology (C). Note the collapse of the peripheral zone between in vivo and ex vivo scans, as well as the distortion around the urethra in the histological section.

with +10 and $-10 \%$ adjustments to the axial plane scale and to fit the resected specimen to the mold that provides the best fit.

- In vivo-ex vivo volume changes. In our experience, there may be significant prostate volume reductions upon resection (see Figure 5) due to loss of luminal fluid. This may lead to a loose and unconstrained fit of the prostate in the mold and consequent misalignment of imaging planes and tissue sectioning planes. Inclusion of the urethral catheter reduces the likelihood of such misalignment, although introducing a small distortion of the tissue around the catheter. As the majority of tumors are not proximal to the urethra, the latter is a relatively minor issue.

- Volume changes due to fixation. The mold constrains prostate movement during fixation, but does not account for volume shrinkage. It is possible that the orientation of the prostate within the mold could change due to the less close fit after shrinkage. This issue could potentially be addressed by transferring the fixed prostate to a second mold with reduced prostate volume; however, we have not implemented this step that assumes a predictable and uniform shrinkage.

- Inconsistent MRI and histology section thickness. The apparatus described improves the reliability of imaging plane alignment with histology planes but does not specifically

\section{REFERENCES}

1. Graham J, Kirkbride P, Cann K, Hasler E, Prettyjohns M. Prostate cancer: summary of updated NICE guidance. BMJ (2014) 348:f7524. doi:10.1136/bmj. f7524

2. Panagiotaki E, Chan RW, Dikaios N, Ahmed HU, O'Callaghan J, Freeman A, et al. Microstructural characterization of normal and malignant human prostate tissue with vascular, extracellular, and restricted diffusion for cytometry in tumours magnetic resonance imaging. Invest Radiol (2015) 50(4):218-27. doi:10.1097/RLI.0000000000000115

3. Wang S, Peng Y, Medved M, Yousuf AN, Ivancevic MK, Karademir I, et al. Hybrid multidimensional T2 and diffusion-weighted MRI for prostate cancer detection. JMagn Reson Imaging (2014) 39(4):781-8. doi:10.1002/jmri. 24212

4. Meyer C, Ma B, Kunju LP, Davenport M, Piert M. Challenges in accurate registration of 3-D medical imaging and histopathology in primary address the problem of sparse histology data in the direction orthogonal to the sectioning planes. This is primarily a tissue processing issue and if resources permit can be addressed by more comprehensive thin sectioning and processing of the tissue blocks.

\section{AUTHOR CONTRIBUTIONS}

Apparatus design: RB. Specimen preparation and handling: GS, $\mathrm{AS}, \mathrm{CB}, \mathrm{SH}, \mathrm{HP}$, and AF. Imaging: $\mathrm{EJ}, \mathrm{CB}$, and BS. Image registration: TM and CB. Project management: DA, DH, SP, and EP. Manuscript preparation: all.

\section{FUNDING}

$\mathrm{RB}$ received research funding from the Australian National Health and Medical Research Council (Grant 1026467). This project was funded by the EPSRC (EP/M020533/1). EP and CB are supported by the EPSRC (EP/N021967/1). HW is supported by Prostate Cancer UK grant PG14-014. HP and EJ by PG14-018-TR2. SH is funded by the Centre of Excellence (CEO13_2-002). The project also received funding from the National Institute for Health Research University College London Hospitals Biomedical Research Centre. prostate cancer. Eur J Nucl Med Mol Imaging (2013) 40(1):72-8. doi:10.1007/ s00259-013-2382-2

5. Orczyk C, Taneja SS, Rusinek H, Rosenkrantz AB. Assessment of change in prostate volume and shape following surgical resection through co-registration of in-vivo MRI and fresh specimen ex-vivo MRI. Clin Radiol (2014) 69(10):e398-403. doi:10.1016/j.crad.2014.06.012

6. Elen A, Isebaert S, De Keyser F, Himmelreich U, Joniau ST, Dresselaers T, et al. Validation and workflow of an improved patient-specific mold design for registration of in-vivo MRI and histology of the prostate. Clinical ImageBased Procedures. Translational Research in Medical Imaging. 5th International Workshop, CLIP 2016, Held in Conjunction with MICCAI 2016, Athens, Greece, October 17, 2016 Proceedings. Athens (2016). p. 36-43.

7. Orczyk C, Mikheev A, Rosenkrantz A, Melamed J, Taneja SS, Rusinek H. Imaging of prostate cancer: a platform for 3D co-registration of in-vivo MRI ex-vivo MRI and pathology. SPIE Medical Imaging. Orlando: International Society for Optics and Photonics (2012). 83162M p. 
8. Bart S, Mozer P, Hemar P, Lenaour G, Comperat E, Renard-Penna R, et al. MRI-histology registration in prostate cancer. In: Merloz P, Troccaz J, editors. Proceedings of Surgetica. Paris: Sauramps Medical (2005). p. 361-7.

9. Kalavagunta C, Zhou X, Schmechel SC, Metzger GJ. Registration of in vivo prostate MRI and pseudo-whole mount histology using local affine transformations guided by internal structures (LATIS). J Magn Reson Imaging (2015) 41(4):1104-14. doi:10.1002/jmri.24629

10. Chappelow J, Bloch BN, Rofsky N, Genega E, Lenkinski R, DeWolf W, et al. Elastic registration of multimodal prostate MRI and histology via multiattribute combined mutual information. Med Phys (2011) 38(4):2005-18. doi:10.1118/1.3560879

11. Xiao G, Bloch BN, Chappelow J, Genega EM, Rofsky NM, Lenkinski RE, et al. Determining histology-MRI slice correspondences for defining MRI-based disease signatures of prostate cancer. Comput Med Imaging Graph (2011) 35(7):568-78. doi:10.1016/j.compmedimag.2010.12.003

12. Shah V, Pohida T, Turkbey B, Mani H, Merino M, Pinto PA, et al. A method for correlating in vivo prostate magnetic resonance imaging and histopathology using individualized magnetic resonance-based molds. Rev Sci Instrum (2009) 80(10):104301. doi:10.1063/1.3242697

13. Trivedi H, Turkbey B, Rastinehad AR, Benjamin CJ, Bernardo M, Pohida T, et al. A step towards personalized medicine: use of a patient-specific MRI based prostate mold for validation of multi-parametric MRI in the localization of prostate cancer. Urology (2012) 79(1):233. doi:10.1016/j.urology.2011.10.002

14. Priester A, Natarajan S, Le JD, Garritano J, Radosavcev B, Grundfest W, et al. A system for evaluating magnetic resonance imaging of prostate cancer using patient-specific 3D printed molds. Am J Clin Exp Urol (2014) 2(2):127.

15. Turkbey B, Mani H, Shah V, Rastinehad AR, Bernardo M, Pohida T, et al. Multiparametric 3T prostate magnetic resonance imaging to detect cancer: histopathological correlation using prostatectomy specimens processed in customized magnetic resonance imaging based molds. JUrol (2011) 186(5):1818-24. doi:10.1016/j.juro.2011.07.013
16. Xu J, Humphrey PA, Kibel AS, Snyder AZ, Narra VR, Ackerman JJ, et al. Magnetic resonance diffusion characteristics of histologically defined prostate cancer in humans. Magn Reson Med (2009) 61(4):842-50. doi:10.1002/ mrm.21896

17. Jonmarker S, Valdman A, Lindberg A, Hellström M, Egevad L. Tissue shrinkage after fixation with formalin injection of prostatectomy specimens. Virchows Arch (2006) 449(3):297-301. doi:10.1007/s00428-006-0259-5

18. Bourne RM, Bongers A, Chatterjee A, Sved P, Watson G. Diffusion anisotropy in fresh and fixed prostate tissue ex vivo. Magn Reson Med (2015) 76(2):626-34. doi:10.1002/mrm.25908

19. Mertzanidou T, Hipwell J, Dalmis M, Platel B, van der Laak J, Mann R, et al. Towards spatial correspondence between specimen and in-vivo breast imaging. In: Fujita H, Hara T, Muramatsu C, editors. Breast Imaging: 12th International Workshop, IWDM 2014, Gifu City, Japan, June 29-July 2, 2014. Proceedings. Gifu: Springer International Publishing (2014). p. 674-80.

20. Ourselin S, Roche A, Subsol G, Pennec X, Ayache N. Reconstructing a 3D structure from serial histological sections. Image Vis Comput (2001) 19(1):25-31. doi:10.1016/S0262-8856(00)00052-4

Conflict of Interest Statement: The authors declare that the research was conducted in the absence of any commercial or financial relationships that could be construed as a potential conflict of interest.

Copyright (C) 2017 Bourne, Bailey, Johnston, Pye, Heavey, Whitaker, Siow, Freeman, Shaw, Sridhar, Mertzanidou, Hawkes, Alexander, Punwani and Panagiotaki. This is an open-access article distributed under the terms of the Creative Commons Attribution License (CC BY). The use, distribution or reproduction in other forums is permitted, provided the original author(s) or licensor are credited and that the original publication in this journal is cited, in accordance with accepted academic practice. No use, distribution or reproduction is permitted which does not comply with these terms. 